\title{
Quality of Life After Open Surgical versus Endovascular Repair of Abdominal Aortic Aneurysms
}

\author{
Mustafa Akbulut ${ }^{1}$, MD; Eray Aksoy ${ }^{1}$, MD; İbrahim Kara² ${ }^{2}$ MD; Davut Cekmecelioglu'1', MD; Cengiz Koksal ${ }^{3}$, MD
}

DOI: 10.21470/1678-9741-2017-0236

\begin{abstract}
Objective: This study aims to compare open surgical and endovascular aneurysm repair (EVAR) of abdominal aortic aneurysms in terms of their effects on quality of life, using Short Form-36 (SF-36).

Methods: A total of 133 consecutive patients who underwent EVAR or open surgical repair for infra-renal abdominal aorta aneurysm between January 2009 and June 2014 were included in the study. Twenty-six (19.5\%) patients died during follow-up and were excluded from the analysis. Overall, 107 patients, 39 $(36.4 \%)$ in the open repair group, and $68(63.6 \%)$ in the EVAR group, completed all follow-up visits and study assessments. Quality of life assessments using SF-36 were performed before surgery and at post-operative months 1, 6, and 12 .
\end{abstract}

Results: The mean duration of follow-up was $29.55 \pm 19.95$ months. At one month, both physical and mental domains of the quality of life assessments favored EVAR, while the two surgical approaches did not differ significantly at or after six months postoperatively.

Conclusion: Despite anatomical advantages and acceptable mid-phase mortality in patients with high- or medium-risk for open surgery, EVAR did not exhibit a quality of life superiority over open surgery in terms of physical function and patient comfort at or after postoperative six months.

Keywords: Quality of Life. Endovascular Procedures. Aortic Aneurysm, Abdominal.

\section{Abbreviations, acronyms \& symbols}

$\begin{array}{ll}\text { AAA } & =\text { Abdominal aortic aneurysm } \\ \text { BP } & =\text { Somatic pain } \\ \text { EVAR } & =\text { Endovascular aneurysm repair } \\ \text { GH } & =\text { General health assessment } \\ \text { MH } & =\text { Mental health } \\ \text { PF } & =\text { Physical function } \\ \text { RE } & =\text { Emotional problems } \\ \text { RP } & =\text { Physical problems } \\ \text { SF } & =\text { Social function } \\ \text { SF-36 } & =\text { Short Form-36 } \\ \text { VT } & =\text { Energy/vitality } \\ \text { WHO } & =\text { World Health Organization }\end{array}$

'Department of Cardiovascular Surgery, Kartal Koşuyolu Research and Training Hospital, Kartal, Istanbul, Turkey.

2Department of Cardiovascular Surgery, Sakarya University Medical Faculty, Sakarya, Turkey.

${ }^{3}$ Department of Cardiovascular Surgery, Bezmialem Vakıf University Medical Faculty, Istanbul, Turkey.

This study was carried out at the Department of Cardiovascular Surgery, Kartal Koşuyolu Research and Training Hospital, Kartal, Istanbul, Turkey.

\section{INTRODUCTION}

Endovascular aneurysm repair (EVAR) is a minimally invasive procedure that was originally developed to reduce the surgical stress levels in patients with a high risk for open surgical repair of abdominal aortic aneurysm (AAA) treatment. Comprehensive studies comparing success rates and outcomes in EVAR and open surgery revealed certain advantages of EVAR over open surgery including reduced blood loss, need for transfusions, need for mechanical ventilation, procedure duration, and intensive care as well as hospital stay ${ }^{[1-4]}$. A further benefit of EVAR was represented by the lower early mortality rate when compared with the open approach. Accordingly, the reported 30-day mortality rates in EVAR groups were $2.1 \%$ in EVAR-1 and $1.2 \%$ in DREAM trials, as compared to the respective figures of $6.2 \%$ and $4.6 \%$ for open surgery; however, this early mortality advantage

No financial support.

No conflict of interest.

Correspondence Address:

Cengiz Köksal

Bezmialem Vakıf University Dragos Hospital

Yalı Mah, Sahil Yolu Sk, No:16, Maltepe, Istanbul, Turkey

E-mail: cengizkoksal@hotmail.com

Article received on December $10^{\text {th }}, 2017$. Article accepted on January 23rd, 2018. 
of EVAR faded over time as a result of subsequent increase in mortality and complications requiring intervention ${ }^{[1,5,6]}$.

Although mortality and morbidity were the key criteria for evaluating the technical success rates of these procedures, health is a "state of complete physical, mental, and social wellbeing, and not merely the absence of disease" as defined by the World Health Organization (WHO). Therefore, the therapeutic approaches should not only aim at prolonging life, but also improving the quality of life. When choosing among therapeutic alternatives, health quality and patient comfort associated with a certain procedure should also be given a consideration in addition to risk assessments. Thus, the present study compared these two surgical repair methods for abdominal aortic aneurysms with respect to their effects on the quality of life.

\section{METHODS}

The target sample population for this single-center study included 133 consecutive patients who underwent aortic aneurysm repair due to infra-renal abdominal aortic aneurysm between January 2009 and June 2014. There were 26 cases of mortality during the follow-up period. Therefore, a total of 107 patients who completed all follow-up assessments were included in the study analyses. Of these patients, 39 (36.4\%) and 68 (63.6\%) were in the surgical repair and EVAR groups, respectively. The mean duration of follow up was $29.55 \pm 19.95$ months (range: 1-78 months). Prior to study procedures, study protocol was approved by the institutional ethics board.

Turkish version of Short Form 36 (SF-36) was used for the evaluation of the quality of life. Validity and reliability of Turkish version has been previously shown ${ }^{[7]}$. In summary, SF-36 is a 36-item, patient-reported outcome measure divided into 8 subscales in 2 domains, i.e. physical and mental health. The physical health domain evaluates physical function (PF), role constraints due to physical problems (RP), somatic pain (BP) and general health assessment (GH), while the mental health domain assesses social function (SF), role constraints due to emotional problems (RE), mental health (MH), energy/vitality (VT). The scale assesses the health status within the past four-week period.

All participants completed SF-36 preoperatively as well as at postoperative months 1,6 , and 12 . For the purpose of the study analyses, scores were evaluated using the coefficients calculated for Turkish standards.

\section{Statistical Analysis}

IBM SPSS Statistics 22 (IBM SPSS, Turkey) software was used for statistical analyses. Shapiro Wilks test was used to test the normality of the data. In addition to descriptive statistics (mean, standard deviation, frequency), Student's t-test was used for comparing quantitative data with normal distribution between the two groups, while Mann-Whitney $U$ test was used for the comparison of data without normal distribution. Within group, comparisons for parameters without normal distribution were performed using Wilcoxon Sign Test. For the comparison of qualitative data, Chi-Square test, Fisher's Exact test, and Yate's Continuity Correction were used. Significance was set at a P level of less than 0.05 .

\section{RESULTS}

Clinical characteristics of the patients in study groups are shown in Table 1. The two groups, i.e. endovascular vs. open surgical repair groups, were comparable in terms of demographic and clinical characteristics $(P>0.05)$, except for more frequent low ejection fraction $(<40 \%)$ in the EVAR group $(P=0.013)$. The mortality rates were $29.09 \%(n=16)$ and $12.82 \%(n=10)$ for open surgical and EVAR groups, respectively. The changes in composite physical and mental scores over time in study groups are shown in Figure 1, 2, and Table 2. Preoperatively, SF-36 scores in the two study groups were similar $(P>0.05)$.

At postoperative month 1, patients in EVAR group had significantly higher (i.e. better) SF-36 scores as compared to those in the open surgery group in all 8 sub-score domains $(P<0.01)$. However, this difference in mental and physical health domain scores between the two groups disappeared at months 6 and $12(P>0.05)$.

\section{DISCUSSION}

Previous trials comparing open surgical repairvs. endovascular stent grafting for the management of abdominal aneurysms in terms of medium and long-term mortality and morbidity rates have provided comparable outcomes for the two approaches ${ }^{[1,5,6]}$. On the other hand, EVAR offered certain morbidity advantages such as reduced need for blood transfusions as well as shortened intensive care and hospital stay ${ }^{[8-10]}$.

EVAR is preferred in high risk or elderly patients, or in those with comorbid conditions based on the minimally invasive nature of the procedure, especially when anatomically feasible. The success of EVAR or open surgical repair for the treatment of abdominal aortic aneurysms has been evaluated through extensive clinical research focusing mainly on mortality and morbidity rates ${ }^{[1,5,6]}$. However, improved life expectancy may represent only one dimension of a treatment effect, which also has an impact on the psychological well-being as well as the patient comfort. Therefore, in this study, two different surgical approaches for the management of abdominal aortic aneurysms were compared with regard to their effects on the quality of life using SF-36, in order to assist the decision-making process prior to surgery in such patients.

SF-36 has been validated as a reliable tool for assessing well-being and health perception from patients' viewpoint. Specifically, vascular surgery societies also have been endorsing the use of SF-36 for follow-up life quality assessments of patients undergoing vascular surgery ${ }^{[1]}$.

In a study by Malina et al. ${ }^{[12]}$, despite lower physical health scores in the first 30 days after surgery among patients undergoing open surgery as compared to those undergoing EVAR, this difference favoring EVAR disappeared at postoperative month 3. In our study, there was a marked reduction in physical and mental health scores during the first postoperative month among open repair patients, while no statistically significant changes in these scores occurred in EVAR group. As earlier pointed out by Chetter et al. ${ }^{[13]}$, energy/vitality represents the single most important determinant of patients' mood. This suggests that the subjective energy level of the patient may be a primary factor driving mental health 
differences in different surgery groups. In the study by Malina et al. ${ }^{[12]}$, mental health scores among the patients showed an increase above the baseline at 3 months after surgery, coinciding with the completion of recovery phase and removal of the life-threatening situation from patients' point of view. It therefore appears that reduced anxiety correlates with improved quality of life.

Lloyd et al. ${ }^{[14]}$ examining these two surgical methods found that life quality scores returned to baseline at six months in both groups, and equaled at 12 months. In another study, although physical health scores were lower than baseline during the first postoperative month in EVAR patients, both physical and mental scores returned to baseline levels at postoperative months 3 and $12^{[6]}$. Furthermore, in some previous studies quality of life in patients undergoing open surgery was worse compared to the normal population during the early postoperative period, and EVAR was also reported to result in worse outcomes in the longer term ${ }^{[15,16]}$. In our study, the two groups did not significantly differ with respect to quality of life scores at or after 6 months postoperatively.

Based on its minimal invasive nature, endovascular stent graft repair may be expected to positively affect the quality of life in patients. However, the need for close monitoring of the patient for endo-leaks, graft failure, and continued expansion of the aneurysmal sac may have an adverse impact on the quality of life, particularly when one considers the potential requirement for re-surgery with open or endovascular approaches as a result of complications ${ }^{[17]}$. Therefore, the weight of evidence suggests that the initial quality of life advantage of EVAR fades over time ${ }^{[18-}$ 20], consistent with our observations showing no difference in terms of physical and mental health scores between the two arms at or after 6 months postoperatively.

Although our study is prospective in nature, one of its potential limitations is the absence of randomization.

\section{CONCLUSION}

The results of our study show a significant positive effect of EVAR on both physical and mental aspects of health as compared to open aneurysm repair during the early postoperative period. However, this early advantage disappears with longer term follow up and the two methods become indistinguishable with regard to life quality effects. Therefore, endovascular repair may represent a better surgical option in elective cases with high comorbidity.

Table 1. Comparison of the groups with respect to preoperative parameters.

\begin{tabular}{|c|c|c|c|c|}
\hline & & Open repair & EVAR & $P$ \\
\hline Age (year) mean $\pm S D$ & & $67.22 \pm 8.92$ & $67 \pm 9.12$ & 0.891 \\
\hline \multirow{2}{*}{ Gender n,\% } & Female & $6(10.9 \%)$ & $10(12.8 \%)$ & \multirow{2}{*}{0.950} \\
\hline & Male & $49(89.1 \%)$ & $68(87.2 \%)$ & \\
\hline Family history n,\% & & $35(63.6 \%)$ & $45(57.7 \%)$ & 0.610 \\
\hline Smoking n,\% & & $44(80 \%)$ & $65(83.3 \%)$ & 0.792 \\
\hline $\mathrm{BMI}>29 \mathrm{n}, \%$ & & $30(54.5 \%)$ & $43(55.1 \%)$ & 1.000 \\
\hline $\mathrm{HL}(\mathrm{LDL}>100) \mathrm{n}, \%$ & & $34(61.8 \%)$ & $56(71.8 \%)$ & 0.306 \\
\hline $\mathrm{DM} n, \%$ & & $17(30.9 \%)$ & $24(30.8 \%)$ & 1.000 \\
\hline $\mathrm{HT} n, \%$ & & $43(78.2 \%)$ & $69(88.5 \%)$ & 0.174 \\
\hline CRF n,\% & & $13(23.6 \%)$ & $24(30.8 \%)$ & 0.479 \\
\hline $\operatorname{COPD}(\mathrm{FEV}<1) n, \%$ & & $24(43.6 \%)$ & $35(44.9 \%)$ & 1.000 \\
\hline PAD n, $\%$ & & $5(9.1 \%)$ & $8(10.3 \%)$ & 1.000 \\
\hline CAD n, $\%$ & & $29(52.7 \%)$ & $49(62.8 \%)$ & 0.244 \\
\hline $\mathrm{EF}<40 \mathrm{n}, \%$ & & $4(7.3 \%)$ & $20(25.6 \%)$ & 0.013 \\
\hline Carotid artery disease $n, \%$ & & $10(18.2 \%)$ & $16(20.5 \%)$ & 0.911 \\
\hline CVA & & $3(5.5 \%)$ & $4(5.1 \%)$ & 1.000 \\
\hline \multirow{2}{*}{ Aneurysm diameter } & $>5,5-7$ & $30(54.5 \%)$ & $44(56.4 \%)$ & \multirow{2}{*}{0.831} \\
\hline & $>7$ & 25 (45.5\%) & $34(43.6 \%)$ & \\
\hline
\end{tabular}

$\mathrm{BMI}=$ body mass index; $\mathrm{CABG}=$ coronary artery bypass grafting; $\mathrm{CAD}=$ coronary artery disease; COPD=chronic obstructive pulmonary disease; $\mathrm{CRH}=$ chronic renal failure; $\mathrm{CVA}=$ cerebrovascular accident; $\mathrm{DM}=$ diabetes mellitus; $\mathrm{EF}=$ ejection fraction; $\mathrm{HL}=$ hyperlipidemia; $\mathrm{HT}=$ hypertension; $\mathrm{PAD}=$ peripheral arterial disease 


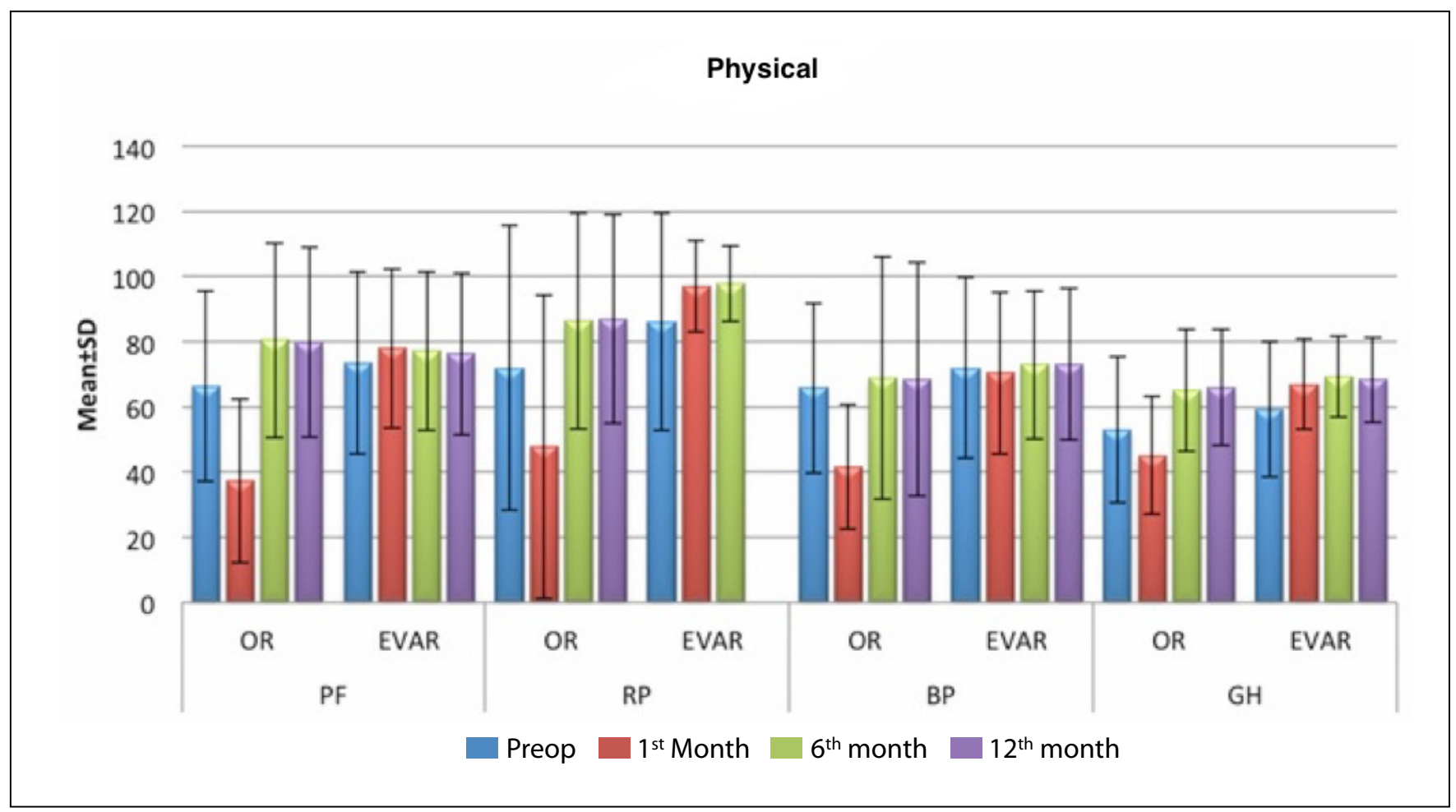

Fig. 1 - Changes in physical domain scores over time.

$B P=$ body pain; $E V A R=$ endovascular aneurysm repair; $G H=$ general health; $O R=$ open repair; $P F=$ physical function; $R P=$ role constraints due to physical problems

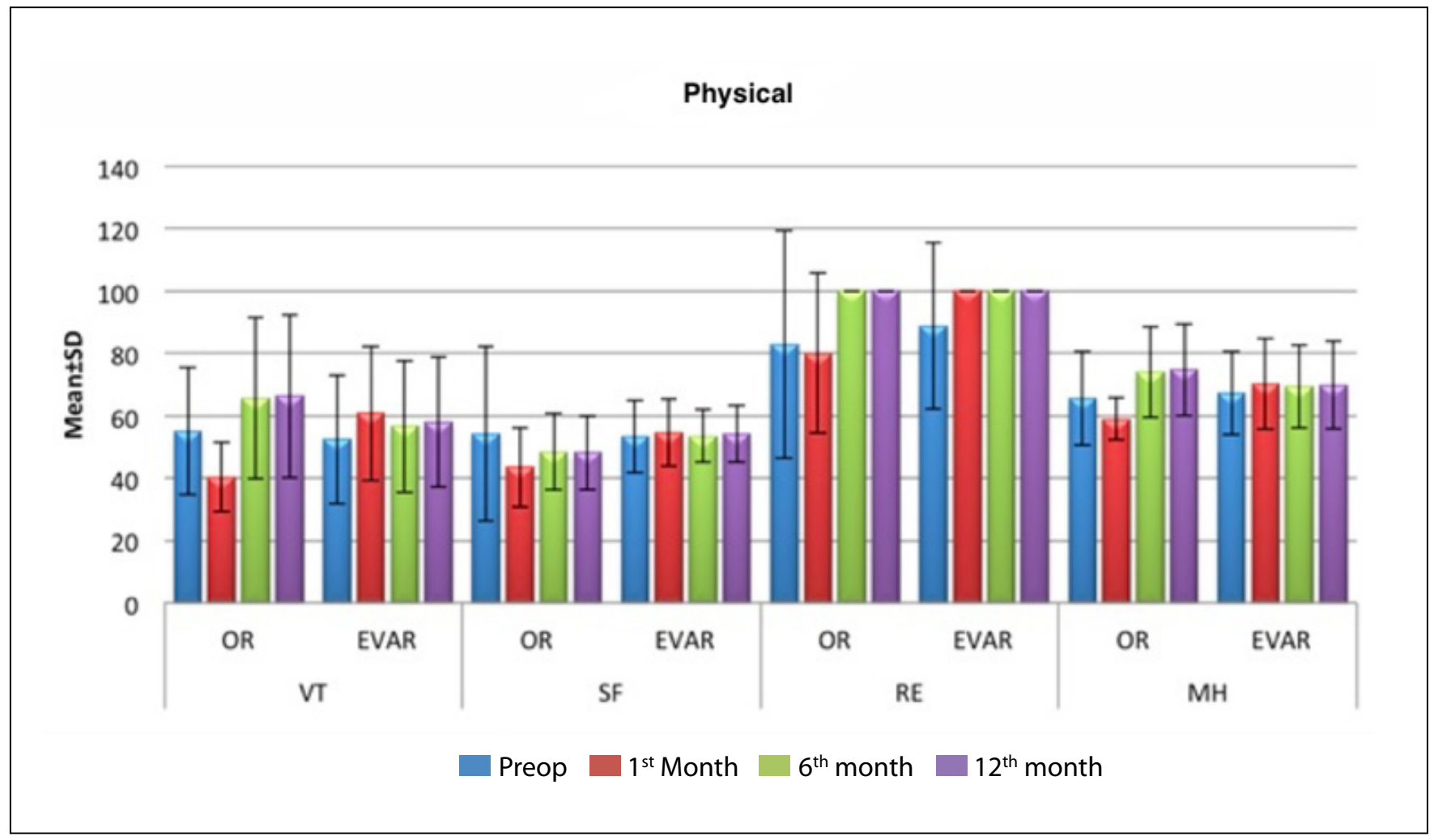

Fig. 2 - Changes in mental domain scores over time.

$E V A R=$ endovascular aneurysm repair; $O R=$ open repair; $M H=$ mental health; $R E=$ role constraints due to emotional problems; $V T=e n e r g y / v i t a l i t y$ 
Table 2. Changes in SF-36 scores over time.

\begin{tabular}{|c|c|c|c|c|c|}
\hline & & Preoperative & $1^{\text {st }}$ month & $6^{\text {th }}$ month & $12^{\text {th }}$ month \\
\hline & & Mean \pm SD (median) & Mean \pm SD (median) & Mean \pm SD (median) & Mean \pm SD (median) \\
\hline \multirow{3}{*}{ PF } & Open repair & $66.36 \pm 29.16(80)$ & $37.27 \pm 25.07(35)$ & $80.48 \pm 29.89(100)$ & $79.85 \pm 29.09(90)$ \\
\hline & EVAR & $73.56 \pm 27.98(90)$ & $77.97 \pm 24.43(95)$ & $77.07 \pm 24.26(92.5)$ & $76.25 \pm 24.86(92.5)$ \\
\hline & ${ }^{1} p$ & 0.112 & $0.001^{* *}$ & 0.314 & 0.410 \\
\hline \multirow{3}{*}{$\mathrm{RP}$} & Open repair & $71.97 \pm 43.64(100)$ & $47.73 \pm 46.5(25)$ & $86.36 \pm 33.13(100)$ & $87.04 \pm 32.05(100)$ \\
\hline & EVAR & $86.02 \pm 33.24(100)$ & $97.03 \pm 13.99(100)$ & $97.83 \pm 11.58(100)$ & $97.83 \pm 11.58(100)$ \\
\hline & ${ }^{1} P$ & 0.128 & $0.001^{* *}$ & $0.040^{*}$ & 0.072 \\
\hline \multirow{3}{*}{ BP } & Open repair & $65.82 \pm 26.05(72)$ & $41.7 \pm 19.14(42)$ & $68.85 \pm 37.13(100)$ & $68.52 \pm 35.78(74)$ \\
\hline & EVAR & $71.98 \pm 27.75(80)$ & $70.42 \pm 24.85(74)$ & $72.87 \pm 22.65(74)$ & $73.18 \pm 23.24(74)$ \\
\hline & ${ }^{1} p$ & 0.318 & $0.001^{* *}$ & 0.652 & 0.903 \\
\hline \multirow{3}{*}{$\mathrm{GH}$} & Open repair & $53.03 \pm 22.41(57)$ & $45.03 \pm 18.07(35)$ & $64.97 \pm 18.59(72)$ & $66 \pm 17.8(72)$ \\
\hline & EVAR & $59.34 \pm 20.77(62)$ & $66.81 \pm 13.84(67)$ & $69.17 \pm 12.38(72)$ & $68.29 \pm 13.04(72)$ \\
\hline & $1 p$ & 0.192 & $0.001^{* *}$ & 0.648 & 0.965 \\
\hline \multirow{3}{*}{ VT } & Open repair & $55 \pm 20.31(55)$ & $40.3 \pm 11.04(45)$ & $65.61 \pm 25.76(75)$ & $66.3 \pm 26.11(75)$ \\
\hline & EVAR & $52.46 \pm 20.67(45)$ & $60.76 \pm 21.58(60)$ & $56.63 \pm 21.16(47.5)$ & $58.04 \pm 20.83(50)$ \\
\hline & ${ }^{1} p$ & 0.422 & $0.001^{* *}$ & 0.072 & 0.133 \\
\hline \multirow{3}{*}{ SF } & Open repair & $54.17 \pm 27.89(62.5)$ & $43.45 \pm 12.84(37.5)$ & $48.48 \pm 12.4(50)$ & $48.15 \pm 11.86(50)$ \\
\hline & EVAR & $53.38 \pm 11.72(50)$ & $54.64 \pm 10.77(50)$ & $53.48 \pm 8.46(50)$ & $54.13 \pm 9.01(50)$ \\
\hline & ${ }^{1} p$ & 0.321 & $0.001^{* *}$ & 0.217 & 0.188 \\
\hline \multirow{3}{*}{$\mathrm{RE}$} & Open repair & $82.83 \pm 36.44(100)$ & $80.1 \pm 25.61(100)$ & $100 \pm 0(100)$ & $100 \pm 0(100)$ \\
\hline & EVAR & $88.89 \pm 26.7(100)$ & $100 \pm 0(100)$ & $100 \pm 0(100)$ & $100 \pm 0(100)$ \\
\hline & $1 P$ & 0.509 & $0.001^{* *}$ & 1 & 1 \\
\hline \multirow{3}{*}{$\mathrm{MH}$} & Open repair & $65.45 \pm 15.03(64)$ & $59.03 \pm 6.93(60)$ & $74.06 \pm 14.7(72)$ & $74.67 \pm 14.63(72)$ \\
\hline & EVAR & $67.12 \pm 13.25(68)$ & $70.1 \pm 14.52(68)$ & $69.3 \pm 13.25(64)$ & $69.86 \pm 14.05(64)$ \\
\hline & ${ }^{1} p$ & 0.733 & $0.001^{* *}$ & 0.091 & 0.173 \\
\hline
\end{tabular}

$\mathrm{BP}=$ body pain; $\mathrm{EVAR}=$ endovascular aneurysm repair; $\mathrm{GH}=$ general health; $\mathrm{PF}=$ physical function; $\mathrm{MH}=$ mental health; $\mathrm{RE}=$ role constraints due to emotional problems; $\mathrm{RP}=$ role constraints due to physical problems; $\mathrm{SF}=$ social function; $\mathrm{VT}=$ energy $/$ vitality; ${ }^{* *} \mathrm{P}<0.05$

\section{Authors' roles \& responsibilities}

MA Substantial contributions to the conception or design of the work; or the acquisition, analysis, or interpretation of data for the work; final approval of the version to be published

EA Drafting the work or revising it critically for important intellectual content; final approval of the version to be published

IK

Agreement to be accountable for all aspects of the work in ensuring that questions related to the accuracy or integrity of any part of the work are appropriately investigated and resolved; final approval of the version to be published

DC Drafting the work or revising it critically for important intellectual content; final approval of the version to be published

CK Final approval of the version to be published

\section{REFERENCES}

1. United Kingdom Evar Trial Investigators, Greenhalgh RM, Brown LC, Powell JT, Thompson SG, Epstein D, Sculpher MJ. Endovascular versus open repair of abdominal aortic aneurysm. $\mathrm{N}$ Engl J Med. 2010;362(20):1863-71.

2. Lederle FA, Freischlag JA, Kyriakides TC, Padberg FT Jr, Matsumura JS, Kohler TR, et al. Outcomes following endovascular vs open repair of abdominal aortic aneurysm: a randomized trial. JAMA. 2009;302(14):1535-42

3. Brewster DC, Geller SC, Kaufman JA, Cambria RP, Gertler JP, LaMuraglia $\mathrm{GM}$, et al. Initial experience with endovascular aneurysm repair: comparison of early results with outcome of conventional open repair. J Vasc Surg. 1998;27(6):992-1003.

4. May J, White GH, Yu W, Ly CN, Waugh R, Stephen MS, et al. Concurrent comparison of endoluminal versus open repair in the treatment of abdominal aortic aneurysms: analysis of 303 patients by life table method. JVasc Surg. 1998;27(2):213-20. 
5. De Bruin JL, Baas AF, Buth J, Prinssen M, Verhoeven EL, Cuypers PW, et al; DREAM Study Group. Long-term outcome of open or endovascular repair of abdominal aortic aneurysm. N Engl J Med. 2010;362(20):1881-9.

6. EVAR trial participants. Endovascular aneurysm repair versus open repair in patients with abdominal aortic aneurysm (EVAR trial 1): randomised controlled trial. Lancet. 2005;365(9478):2179-86.

7. Koçyiğit H, Aydemir Ö, Fişek G, Ölmez N, Memiş A. Kısa Form-36 (KF36)'nın Türkçe versiyonunun güvenilirliği ve geçerliliği: romatizmal hastalığı olan bir grup hasta ile çalışma. Illaç ve Tedavi Dergisi. 1999;12:102-6.

8. Leurs $L J$, Buth J, Laheij RJ. Long-term results of endovascular abdominal aortic aneurysm treatment with the first generation of commercially available stent grafts. Arch Surg. 2007;142(1):33-41.

9. Moore WS, Kashyap VS, Vescera CL, Quiñones-Baldrich WJ. Abdominal aortic aneurysm: a 6-year comparison of endovascular versus transabdominal repair. Ann Surg. 1999;230(3):298-306.

10. May J, White GH, Waugh R, Ly CN, Stephen MS, Jones MA, et al. Improved survival after endoluminal repair with second-generation prostheses compared with open repair in the treatment of abdominal aortic aneurysms: a 5-year concurrent comparison using life table method. J Vasc Surg. 2001;33(2 Suppl):S21-6.

11. McDaniel MD, Nehler MR, Santilli SM, Hiatt WR, Regensteiner JG, Goldstone J, et al. Extended outcome assessment in the care of vascular diseases: revising the paradigm for the 21st century. Ad Hoc Committee to Study Outcomes Assessment, Society for Vascular Surgery/International Society for Cardiovascular Surgery, North American Chapter. J Vasc Surg. 2000;32(6):1239-50.

12. Malina M, Nilsson M, Brunkwall J, Ivancev K, Resch T, Lindblad B. Quality of life before and after endovascular and open repair of asymptomatic
AAAs: a prospective study. J Endovasc Ther. 2000;7(5):372-9.

13. Chetter IC, Spark JI, Dolan P, Scott DJ, Kester RC. Quality of life analysis in patients with lower limb ischaemia: suggestions for European standardisation. Eur J Vasc Endovasc Surg. 1997;13(6):597-604.

14. Lloyd AJ, Boyle J, Bell PR, Thompson MM. Comparison of cognitive function and quality of life after endovascular or conventional aortic aneurysm repair. Br J Surg. 2000;87(4):443-7.

15. Peach G, Holt P, Loftus I, Thompson MM, Hinchliffe R. Questions remain about quality of life after abdominal aortic aneurysm repair. J Vasc Surg. 2012;56(2):520-7.

16. Prinssen M, Buskens E, Blankensteijn JD; DREAM trial participants. Quality of life endovascular and open AAA repair. Results of a randomised trial. Eur J Vasc Endovasc Surg. 2004;27(2):121-7.

17. Thompson MM, Boyle JR, Hartshorn T, Maltezos C, Nasim A, Sayers RD, et al. Comparison of computed tomography and duplex imaging in assessing aortic morphology following endovascular aneurysm repair. Br J Surg. 1998;85(3):346-50.

18. Coughlin PA, Jackson D, White AD, Bailey MA, Farrow C, Scott DJ, et al. Meta-analysis of prospective trials determining the short-and mid-term effect of elective open and endovascular repair of abdominal aortic aneurysms on quality of life. Br J Surg. 2013;100(4):448-55.

19. de Bruin JL, Groenwold RH, Baas AF, Brownrigg JR, Prinssen M, Grobbee DE; DREAM Study Group. Quality of life from a randomized trial of open and endovascular repair for abdominal aortic aneurysm. Br J Surg. 2016;103(8):995-1002.

20. Tomita TM, Hoel AW. Does EVAR improve outcomes or quality of life in patients unfit for open surgery?. In: Skelly C, Milner R, eds. Difficult decisions in vascular surgery: an evidence-based approach. Cham: Springer International Publishing; 2017. p.87-97. 